\title{
El Community Manager en las principales empresas de España: una aproximación a su formación y su situación laboral
}

\author{
Ana Almansa MartíneZ \\ Universidad de Málaga \\ anaalmansa@uma.es \\ Francisco Javier GODOY MARTíN \\ Universidad de Málaga \\ frangm@gmail.com
}

Recibido: 31/07/2012

Aceptado: 11/10/2012

\begin{abstract}
Resumen
El número de empresas que desarrollan su presencia en medios sociales es cada vez mayor. Como consecuencia, han aparecido nuevos perfiles profesionales. El Community Manager es el más conocido actualmente. El principal objetivo de esta investigación es analizar la profesión de Community Manager en las principales empresas de España. Por ello, a través de una metodología descriptiva de carácter cuantitativo basada en la encuesta, se pretende obtener datos acerca de la situación laboral de estos profesionales, así como de su formación y su relación con el campo de la comunicación y las relaciones públicas. Los resultados reflejan que se trata de empleados con cierta estabilidad y antigüedad en su empresa, vinculados al campo de la comunicación, especialmente el periodismo, que se han sometido a un proceso de reciclaje.
\end{abstract}

Palabras clave: Community Manager, Internet, Relaciones Públicas, Web 2.0

\section{The Community Manager in leading companies in Spain: an approach to their training and employment situation}

\begin{abstract}
The number of companies developing their presence in Social Media is growing. As a consequence, new professional profiles have appeared. Nowadays the Community Manager is the most known. This research is aimed at analysing the profession of Community Manager in leading companies in Spain. Therefore, following a quantitative descriptive methodology based on the survey, this research aims to obtain data about the employment situation of these professionals as well as their training and their connection to the field of communication and public relations. The results obtained show that they are employees with a certain stability and seniority in their companies, linked to the field of communication, especially journalism, and who have undergone a process of retraining.
\end{abstract}

Keywords: Community Manager, Internet, Public Relations, Web 2.0

\section{Referencia normalizada}

ALMANSA MARTÍNEZ, Ana y GODOY MARTÍN, Francisco Javier (2012): "El Community manager en las principales empresas de España: una aproximación a su formación y su situación laboral". Estudios sobre el mensaje periodístico. Vol. 18, núm. especial octubre, págs.: 57-65. Madrid, Servicio de Publicaciones de la Universidad Complutense.

Sumario: 1. Introducción: las empresas y los medios sociales. 2. Gestión de comunidades online y relaciones públicas. 3. Metodología. 4. Resultados de la investigación. 5. Conclusiones. 6. Referencias bibliográficas. 


\section{Introducción: las empresas y los medios sociales}

Internet se ha convertido en un medio cada vez más interesante para las empresas, sobre todo teniendo en cuenta que su audiencia ha pasado del $10,6 \%$ al $44,7 \%$ en la última década, situándose como el medio que registra una mayor subida en este período (AIMC, 2012b: 3).

Si bien a lo largo de las últimas décadas han existido iniciativas que perseguían facilitar las relaciones entre personas (Marín de la Iglesia, 2010), es en la actualidad, con el desarrollo de la Web 2.0, cuando parece que la participación y la interacción se convierten en características fundamentales de la Red.

Martínez Estremera (2012: 9) define el Social Media como "un grupo de medios de comunicación online" caracterizados por ser participativos y abiertos, permitir conversaciones, crear comunidad y desarrollar conectividad. La cuarta oleada del Observatorio de Redes Sociales de la empresa The Cocktail Analysis señala que durante 2011 se produjo un proceso de "sofisticación" del usuario, que pasa a desempeñar un rol más activo en la gestión de su presencia en medios sociales. Además, "más de la mitad de usuarios de redes sociales y comunidades se muestran a priori de acuerdo con la idea de que su presencia puede ser interesante desde el punto de vista de los contenidos (información en primicia, exclusiva para usuarios de redes...), desde el punto de vista del ahorro (promociones y ofertas...) e incluso, más allá, puede llegar a ser un canal más de servicio de atención al cliente" (The Cocktail Analysis, 2012: 42).

Pero la Web 2.0 no ha supuesto sólo un cambio en el modelo comunicativo, sino también, productivo: "nos encontramos ante la posibilidad de que todas y cada una de las personas del planeta tengan acceso a los mismos contenidos y servicios. O lo que es lo mismo, que cualquier operador, por pequeño que sea, tenga la posibilidad de acceder a una audiencia global para sus contenidos o a un mercado universal de clientes para sus productos" (Marín de la Iglesia, 2010: 34).

En el ámbito comunicativo, esta nueva situación conlleva "una evolución de su identidad, pues el escuchar y, sobre todo, conversar y relacionarse con los stakeholders es una cuestión comprendida, pero el sumergirse en este nuevo reto tecnológicosocial, conlleva una transformación de la cultura corporativa de la empresa" (Mut, 2011: 356).

Parece, por tanto, que en el campo de la comunicación y las relaciones públicas, el modelo al que mejor se adaptaría la Web 2.0 es el simétrico bidireccional, enunciado por Grunig y Hunt (2003), caracterizado por la comprensión y la influencia mutua entre los públicos y la organización. Sin embargo, no hay que olvidar que las empresas son entidades con unos objetivos tanto económicos como comunicativos marcados, por lo que "ya es hora de terminar con la consideración de las relaciones públicas como la comunicación bidireccional simétrica que lo que pretende es el entendimiento mutuo y el consenso entre las organizaciones y sus públicos. Esto no es así porque es utópico; y es utópico porque, hoy y siempre, los profesionales de las relaciones públicas han prestado sus servicios a clientes y organizaciones que les han contratado para defender sus intereses en términos de imagen pública y reputación, es decir, en términos también económicos, pues una empresa necesita tener una reputación positiva únicamente para fomentar sus beneficios económicos" (Xifra, 2011: 19). 
En cualquier caso, lo importante es entender la comunicación y las herramientas utilizadas en ella, incluidos los medios sociales, en un sentido estratégico, llevando a cabo acciones coherentes encaminadas a conseguir unos objetivos fijados previamente. En este sentido, "no hay que potenciar el uso de las tecnologías porque socialmente sea lo correcto, sino porque sea la consecuencia de una decisión meditada" (Castillo y Almansa, 2005: 138).

\section{Gestión de comunidades online y relaciones públicas}

A pesar de que existen varios perfiles profesionales relacionados con los medios sociales, actualmente la figura más conocida es la de Community Manager. Es frecuente encontrar en los últimos tiempos numerosos artículos sobre qué es y qué no es un Community Manager y cuáles son sus funciones. La Asociación Española de Responsables de Comunidad y Profesionales Social Media (AERCO-PSM) lo define como "aquella persona encargada o responsable de sostener, acrecentar y, en cierta forma, defender las relaciones de la empresa con sus clientes en el ámbito digital, gracias al conocimiento de las necesidades y los planteamientos estratégicos de la organización y los intereses de los clientes. Una persona que conoce los objetivos y actúa en consecuencia para conseguirlos" (Gallego et al., 2009: 5).

Al pensar en las relaciones públicas se observa una serie de elementos comunes con esta definición. Así, "los publirrelacionistas se comunican con todos los públicos internos y externos relevantes para desarrollar relaciones positivas y crear una consistencia entre las metas de la organización y las expectativas de la sociedad. Los publirrelacionistas desarrollan, ejecutan y evalúan programas de la organización que propician el intercambio de influencia y conocimiento entre las partes que forman una organización y los diferentes públicos" (Lattimore et al., 2008: 4).

Encontramos, por tanto, puntos comunes entre ambas definiciones (objetivos o metas, relaciones e intercambio entre organizaciones y públicos, intereses y expectativas) que nos remiten al concepto de estrategia y, como consecuencia, al ámbito de las relaciones públicas.

En referencia a las funciones que desempeña un gestor de comunidades online o Community Manager en el marco de una organización, se resumen en cinco tareas básicas: monitorizar y escuchar activamente las conversaciones sobre su organización; hacer circular esta información internamente; saber explicar la posición de la empresa a la comunidad; buscar líderes tanto interna como externamente; y encontrar vías de colaboración entre la comunidad y la empresa (Mencía, 2012: 117).

\section{Metodología}

El principal objetivo de este trabajo es conocer el uso de los medios sociales y el grado de implantación de la profesión de Community Manager en las principales empresas de España, así como determinar su perfil formativo y sus condiciones laborales.

Para alcanzar estos objetivos se ha recurrido a una metodología descriptiva de carácter cuantitativo cuya técnica principal ha sido la encuesta. El universo de estudio de la investigación estaba compuesto por todas las grandes empresas que desarrollan su actividad económica en España. Para llevar a cabo el estudio se ha acotado una mues- 
tra formada por las 35 principales compañías del país, atendiendo a su presencia en el Ibex 35. Esta selección se debe a que estas firmas a priori cuentan con más recursos para implementar acciones de comunicación y, por tanto, en medios sociales. Hay que tener en cuenta también que algunas de estas empresas pueden contar con perfiles en medios sociales en otros idiomas, especialmente inglés, debido a su carácter multinacional. En este caso, centrado en la profesión de Community Manager en España, se han tenido sólo en cuenta aquellas plataformas gestionadas en el país e idioma españoles.

La investigación se ha llevado a cabo en dos fases: una primera orientada a determinar, a través del análisis de contenido de sus páginas web, cuáles de estas empresas tienen presencia en Internet a través de medios sociales; y una segunda consistente en el envío de un cuestionario a sus gestores de comunidades online, a fin de determinar su nivel de formación, titulación y condiciones laborales, así como otras cuestiones relacionadas con la Web 2.0, las relaciones públicas y el uso estratégico de estas herramientas.

\section{Resultados de la investigación}

El análisis de contenido inicial de las páginas web revela que 8 de las 35 empresas del Ibex 35 no utilizan medios sociales, por lo que la muestra final de la investigación queda constituida por 27 empresas, a las que se les envió el cuestionario. De ellas, han contestado 20 , lo que supone un $74,1 \%$ del total, porcentaje considerado válido para obtener resultados para este estudio.

Este análisis refleja, además, que las herramientas 2.0 más utilizadas por las principales empresas de España son Twitter (25 empresas), Facebook (21), YouTube (20), los blogs corporativos (12) y Linkedin (9). También aparecen mencionadas nuevas plataformas como Pinterest y otras que no son tan habituales como las primeras (Scribd, Vimeo, Xing o Picasa).

Según se desprende de la encuesta realizada a los Community Managers de estas empresas, en la actualidad Twitter es la herramienta más valorada para la comunicación externa, marcada en un $100 \%$ de los casos (los usuarios podían elegir varias opciones, por lo que los porcentajes no tienen que sumar 100), seguida de Facebook y YouTube, con un $85 \%$ cada una, y los blogs corporativos, con un $70 \%$. Aparece ya en algún caso Instagram, lo que pone de manifiesto una continua adaptación a las nuevas tecnologías. Sin embargo, las plataformas que son muy valoradas en la comunicación externa no son tan relevantes en el caso de la comunicación interna. Así, Twitter y Facebook reciben un $28,6 \%$ de las valoraciones positivas y YouTube, un 35,7\%. En cambio, los blogs corporativos son considerados la mejor herramienta, marcada en un $92,9 \%$ de los casos. Además, aparecen otras no contempladas inicialmente en la investigación, como Yammer, indicada en un 14,3\% de los casos, o la intranet, que también es considerada como una herramienta colaborativa en el ámbito interno, ya que fue apuntada por el $35,7 \%$ de los participantes.

Por lo general, son los departamentos de prensa y comunicación los que gestionan los medios sociales de estas compañías (en un 70\% de los casos), seguidos de los departamentos de Marketing (15\%) e Informática (5\%). Asimismo, en un 10\% de los casos existen departamentos específicos, como Comunicación Digital o Internet. Ade- 
más, estas tareas son llevadas a cabo por varias personas en el $65 \%$ de las empresas participantes. De hecho, en el 60\% de ellas, existen otros puestos relacionados con Social Media, como Social Media Strategist, Social Media Analyst, Online Reputation Manager o Social Media Manager.

El trabajo del Community Manager está principalmente orientado hacia los públicos externos, ya que un $50 \%$ de los encuestados marcó esta opción, mientras que otro $50 \%$ eligió "Ambos tipos de públicos" y en ningún caso se señaló la opción del ámbito exclusivamente interno. Las labores realizadas principalmente por estos profesionales son "Monitorización de la empresa / Marca y Definición de estrategias 2.0", ambas seleccionadas en el 95\% de los casos; "Monitorización del sector", con un 85\%; y "Monitorización de la competencia, Informes para directivos de la empresa, Redacción de contenidos para redes sociales" y "Gestión de crisis online", con un $80 \%$ cada una. Por otra parte, destaca el amplio porcentaje recibido por la opción "Resolución de quejas y consultas" (75\%). Sin embargo, no son éstas las únicas tareas que llevan a cabo estos profesionales durante su jornada laboral, ya que un $70 \%$ de los encuestados afirma compaginar sus labores con otras funciones dentro de su departamento: redacción de notas de prensa e informes, seguimiento de medios, acciones de marketing o webmaster, por citar algunas.

A pesar de que la mayoría de los encuestados pertenece a departamentos de prensa y comunicación, no hay unanimidad a la hora de enmarcar la gestión de medios sociales en una disciplina concreta. Se les propuso a los profesionales situar esta labor en una escala donde 1 era Relaciones Públicas y 10, Marketing. Las respuestas ofrecen una media de 4,8, concentrándose, además, el 90\% entre los valores comprendidos entre el 3 y el 6 .

No obstante, hay consciencia de que estas herramientas deben ser utilizadas de forma estratégica: al preguntar si las acciones 2.0 son efectivas, un 35\% declaró Sí, siempre, mientras que un $65 \%$ eligió la opción "Depende de diversos factores como objetivos, segmentación, tipo de acción o plataforma utilizada". En ningún caso se marcó No, nunca. Además, en un $90 \%$ de los casos, las acciones 2.0 están incluidas en el plan de comunicación de la empresa y un $95 \%$ declaró que su empresa dispone, además, de un plan específico para Social Media, en cuyo diseño participaron el 85\% de estos profesionales.

Siguiendo con el concepto de estrategia, se les propuso a los participantes la evaluación de la importancia de una serie de factores en una campaña de Social Media. Los resultados muestran que los más valorados, por orden de importancia, son los objetivos $(9,1$ de media), el tipo de plataforma $(8,2)$, la segmentación del público $(8,1)$, el tipo de acción $(7,9)$, los recursos humanos empleados $(7,5)$ y los recursos económicos empleados $(6,9)$.

En cualquier caso, se da por hecho que los medios sociales están plenamente implantados en las rutinas comunicativas de las empresas, ya que el $100 \%$ de los encuestados afirma que la Web 2.0 ha cambiado sustancialmente la forma en que las organizaciones se relacionan con sus públicos.

El análisis del perfil formativo de los Community Managers de las empresas del Ibex 35 refleja que la mayoría de los profesionales tiene un nivel de estudios superior, 
ya sea licenciatura o grado $(40 \%)$, máster $(50 \%)$ u otra formación de postgrado $(5 \%)$. Entre los estudios indicados por los encuestados, destaca que un $80 \%$ pertenece a Comunicación. De esta manera un 60\% son periodistas, frente a un 5\% (un único caso) de Publicidad y Relaciones Públicas y un 10\% de Comunicación Audiovisual. También aparecen otros estudios como Ingeniería de Telecomunicaciones, Empresariales o Administración y Dirección de Empresas.

Además, un $80 \%$ cuenta con formación de postgrado en comunicación. De ellos, un $68,8 \%$ la recibió en centros privados. En lo que se refiere a formación específica en Social Media, un 55\% la recibió, todos también en centros privados. Los precios pagados por estos estudios son considerablemente altos: un $54,5 \%$ entre 1.000 y 3.000 euros; un $18,2 \%$ entre 3.000 y 5.000 euros; y un $9,1 \%$ para cada una de las opciones de menos de 500 euros, entre 500 y 1.000 euros y más de 5.000 euros.

En el plano laboral, nos encontramos con trabajadores con antigüedad en la empresa, ya que el $45 \%$ lleva en ella más de cinco años y un 35\% entre 1 y 5 años. La mayoría $(65 \%)$ no fueron contratados específicamente para desempeñar funciones de Community Manager. De hecho, un 70\% realiza estas tareas desde hace entre 1 y 3 años y un 30\% desde hace menos de un año. En cualquier caso, se trata de profesionales con una importante estabilidad, ya que el $90 \%$ de los encuestados está vinculado a su empresa mediante un contrato indefinido, el $100 \%$ trabaja a jornada completa y el $70 \%$ cobra más de 30.000 euros brutos anuales. Además, un $55 \%$ cree que tiene posibilidades de ascenso, frente a un $20 \%$ que cree que no las tiene y un $25 \%$ que no se lo ha planteado.

Por último, la media de edad de estos profesionales es de 34 años, un $50 \%$ hombres y un $45 \%$ mujeres (un 5\% no contestó). El $85 \%$ de los encuestados era usuario de medios sociales antes de dedicarse a ellos profesionalmente.

\section{Conclusiones}

Los resultados obtenidos en la investigación reflejan que los medios sociales están plenamente implantados en las principales compañías de España, especialmente para la relación con los públicos externos, ya que más de las tres cuartas partes utiliza al menos alguno de estos recursos. No en vano, la totalidad de los encuestados señala que la Web 2.0 ha cambiado considerablemente la forma en que las organizaciones se relacionan con sus públicos. Tampoco hay que olvidar que, además del Community Manager, en muchos casos existen otros puestos relacionados con Social Media, lo que pone de manifiesto la importancia que se está dando actualmente a estos canales, que se han convertido, más que en el futuro, en el presente de las relaciones con los públicos. No es de extrañar entonces que, entre las funciones más habituales indicadas por los profesionales encuestados, ocupe uno de los primeros lugares la resolución de consultas y quejas, manifestando una preferencia por parte de los clientes y usuarios por estos canales para dirigirse a la empresa.

Los elementos comunes entre Community Manager y Relaciones Públicas, apuntados en el marco teórico, se observan también en la práctica cotidiana de la profesión, puesto que las herramientas propias de la Web 2.0 se gestionan, por lo general, desde los departamentos de comunicación y prensa. Sin embargo, llama la atención que los 
profesionales encuestados no las sitúen de una forma clara en la disciplina de las Relaciones Públicas. Parece, por tanto, que se consideran recursos de gran potencial que pueden ser aprovechados por otras áreas de la empresa.

Pero, a pesar de la indefinición a la hora de ubicar los medios sociales en un determinado campo, se observa que es fundamental utilizarlos de forma estratégica, un concepto clave en las Relaciones Públicas. Sólo el hecho de que las mismas plataformas no sean valoradas de la misma manera cuando se trata de comunicación externa que cuando se trabaja con públicos internos apoya esta afirmación. A esto hay que añadir la inclusión de acciones 2.0 en el plan de comunicación y la existencia, además, de un plan específico para Social Media, en cuyo diseño participa el $85 \%$ de estos profesionales, por lo que no se trata de un perfil meramente ejecutivo. Incide en esta idea que la mayoría de los participantes indicara que el éxito de las acciones 2.0 está determinado por diversos factores como objetivos, segmentación o tipo de plataforma, entre otros. La valoración de los recursos económicos en último lugar de importancia en una campaña de Social Media remite a la democratización en el acceso a los diferentes mercados del que disponen las empresas gracias a estas herramientas, No obstante, hay que tener en cuenta que en este estudio se limita a empresas con importantes recursos, lo que puede influir de cierta forma en la despreocupación por lo económico.

El análisis de la formación recibida señala que se trata de profesionales con un alto nivel formativo, relacionados mayoritariamente con el ámbito de la Comunicación, principalmente periodistas, por lo que existe la posibilidad de que no tengan tan presente las Relaciones Públicas, lo que, además, explicaría la indefinición a la hora de posicionar su gestión en uno u otro ámbito. Destaca, por tanto, el bajo porcentaje de Community Managers procedentes de Publicidad y Relaciones Públicas (un solo caso en este estudio).

En el plano laboral, la estabilidad que se desprende de los datos (antigüedad, contrato indefinido, jornada laboral completa, sueldos superiores a 30.000 euros anuales), unida a la compaginación de la gestión de medios sociales con otras tareas dentro de los departamentos y al hecho de que un $65 \%$ no fue contratado específicamente para ejercer de Community Manager, indica que se ha producido un proceso de reciclaje entre los profesionales ya presentes en la empresa, de manera que han adoptado estas funciones a la vez que realizan otras.

Considerando los resultados del estudio, podría afirmarse que el Community Manager de las principales empresas de España es una persona joven, licenciada en Periodismo con formación adicional en Comunicación y en Social Media, con buenas condiciones laborales y cierta antigüedad en su compañía, que se han sometido a un proceso de reciclaje profesional a fin de poder hacer frente a estas nuevas labores.

No obstante, parece claro que la profesión de Community Manager se irá definiendo y consolidando con el tiempo y se convertirá en un puesto cada vez más importante en las organizaciones, ya que supone un trato directo e inmediato con los públicos. Para ello, estos profesionales deberán poseer una alta cualificación en comunicación online y nuevas tecnologías, pero también, y con mayor importancia, en estrategias de comunicación. 


\section{Referencias bibliográficas}

ASOCIACIÓN PARA LA INVESTIGACIÓN DE MEDIOS DE COMUNICACIÓN (2012b): "Resumen general del Estudio General de Medios. Octubre de 2011 a mayo de 2012", en http://www.aimc.es/-Datos-EGM-Resumen-General-.html [fecha de consulta: 21 de agosto de 2012].

CASTILLO ESPARCIA, Antonio y ALMANSA MARTÍNEZ, Ana (2005): "Relaciones públicas y tecnología de la comunicación. Analisys de los sitios de prensa virtuales", en Organicom, n 3, pp. 134-149.

GALLEGO, José Antonio; POLO, Fernando; RÍOS, Palmira; OREJÓN LOZANO, Selva María y GARCÍA-ALBERTOS JIMÉNEZ, María (2009): "La función del Community Manager", en https://www.box.com/shared/pgur4btexi [fecha de consulta: 12 de julio de 2012].

GRUNIG, James E. y HUNT, Todd (2003): Dirección de relaciones públicas. Barcelona, Gestión 2000.

LATTIMORE, Dan; BASKIN, Otis; HEIMAN; Suzette. T. y TOTH, Elizabeth L. (2008): Relaciones públicas. Profesión y práctica. México, McGraw Hill.

MARÍN DE LA IGLESIA, José Luis (2010): Web 2.0: una descripción muy sencilla de los cambios que estamos viviendo. Oleiros (La Coruña), Netbiblo.

MARTÍNEZ ESTREMERA, Juan Ignacio (2012): "Introducción a los medios sociales (Social Media)", en MARTÍNEZ ESTREMERA, Juan Ignacio (coord.): Community Manager: gestión de comunidades virtuales, pp. 8-15 http://www.aercomunidad.org/publicaciones/gestion-de-comunidades-virtuales [fecha de consulta: 16 de agosto de 2012].

MENCÍA, José Manuel (2012): "Habilidades del Community Manager", en MARTÍNEZ ESTREMERA, Juan Ignacio (coord.): Community Manager: gestión de comunidades virtuales, pp.

$115-120$ http://www.aercomunidad.org/publicaciones/gestion-de-comunidades-virtuales [fecha de consulta: 16 de agosto de 2012].

MUT CAMACHO, Magdalena (2011): "Relaciones públicas 2.0: Twitter y la comunicación interna", en Actas del VI Congreso Internacional de Investigación y Relaciones Públicas, pp. 354-367. http://www.icono14.es/vi-ci-rrpp [fecha de consulta: 16 de julio de 2012].

THE COCKTAILANALYSIS (2012): "Observatorio de redes sociales. IV oleada", en http://www.slideshare.net/TCAnalysis/4-oleada-observatorio-de-redes-sociales [fecha de consulta: 16 de agosto de 2012].

XIFRA, Jordi (2011): Manual de relaciones públicas e institucionales. Madrid, Tecnos. 


\section{Ana ALMANSA MARTíNEZ}

Universidad de Málaga

anaalmansa@uma.es

Facultad de Ciencias de la Comunicación

Campus de Teatinos, $\mathrm{s} / \mathrm{n}$

29071 Málaga (España)

Profesora Titular de Universidad, adscrita al Departamento de Comunicación Audiovisual y Publicidad, Facultad de Ciencias de la Comunicación, de la Universidad de Málaga.

\section{Francisco Javier GODOY MARTÍN}

Universidad de Málaga

frangm@gmail.com

Facultad de Ciencias de la Comunicación

Campus de Teatinos, $\mathrm{s} / \mathrm{n}$

29071 Málaga (España)

Doctorando del Doctorado Dirección Estratégica en Comunicación, de la Universidad de Málaga. Ha trabajado en los gabinetes de comunicación de la Universidad Internacional de Andalucía, el Instituto de Estadística de Andalucía, en Ibersponsor, en Grayling, en la División de Comunicación de Extenda-Agencia Andaluza de Promoción Exterior, en Aerospace \& Defense Meetings-ADM Sevilla 2012. 